\title{
Selective Active Filter with Remote Harmonic Distortion Control
}

\author{
Gonzalo Casaravilla, Adriana Salvia, Cesar Briozzo, Edson Watanabe
}

\begin{abstract}
This paper presents the calculation methods for the different filtering blocks of a selective active filter in order to minimize the active filter inverter size, keeping the harmonic emissions at the limits stated by the applicable regulations. Since it may be necessary to comply with the regulations at a remote place with respect to the active filter location, the method has been extended to this case. In order to validate the simulations, measurements which were made at a real application - and arc furnace - were used. Finally, the simulated results obtained with a remote hybrid filter were compared with those obtained when an active filter is installed directly at the place where the harmonic emission regulations must be met. The comparison shows that remote compensation is an alternative to be seriously considered. The results are similar to those obtained with local compensation and the cost may be substantially lower.
\end{abstract}

Index Terms-Harmonics, power quality, active filters.

\section{INTRODUCTION}

One of the main applications of active filters is the elimination or reduction of current and voltage harmonics. $\mathrm{Pq}$ theory (active and imaginary instant power) [1], has been proven as being useful to understand this problem and has simplified its control in real time. Standards and regulations [2] [3] [4] establish that a certain maximum current harmonics emission must not be exceeded by an individual electricity consumer. For each individual harmonic $(2,3,4, \ldots . n)$ a maximum level is established. For the total harmonic distortion (THD) a maximum is also established. This last value is always less than the distortion that would be obtained if the required individual values were exactly satisfied. If the goal is to design a minimum cost active filter, its inverter must be of minimum current [5]. Therefore, individual and total harmonic distortions must not be reduced more than it is required. In [5] the harmonic residue was filtered in an indiscriminate way with results that were not acceptable at all. Then the necessity of selective filtering arises. In this paper an optimization method for this selectivity will be presented, as it was announced in [6] [7].

Different papers [8] [9] [10] [11] [12] show particular examples of selectivity accomplished by using the SRF (Synchronous Reference Frame) calculation strategy, which is equivalent to using pq theory, as shown in [6] [7]. The need of developing SERIES and PARALLEL calculation methods arises as a result of doing the selective filtering of several harmonic frequencies which are close to each other in the frequency spectrum. These calculation methods are presented in [6], where control is achieved by measuring the load current,

G. Casaravilla - llE-UDELAR-Unuguay, gcp@iie.edu.uy A. Salvia - IIE-UDELAR-Uruguay, adriana@iie.edu.uy C. Briozzo - IIE-UDELAR-Uruguay, cesar@iic.edu.uy E. Watanabe · COPPE-UFRJ-Brasil, watanabe@coe.ufj.br

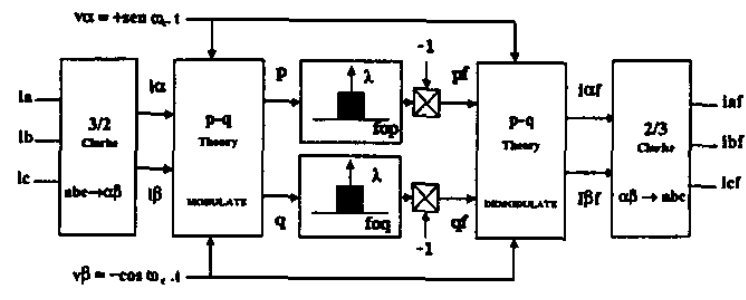

Fig. 1. Modulation - Demodulation with $+w c$

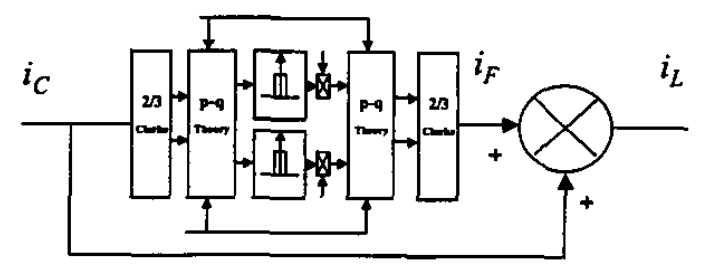

Fig. 2. Load current compensation

and they are generalized in [7] for load current measuring (compensation) or for line current measuring (feedback) [13]. In order to follow the concept 'you dirty, you clean', the best option is the use of a shunt active filter controlled in such a way that it compensates distorted load currents [7]. An hybrid active passive configuration must be adopted when it is necessary to minimize the cost of the active filter or to use already installed passive filters (even improving its tunning)[14] [11] [7]

\section{LOCAL SELECTIVE ACTIVE FILTER}

\section{A. Selective filtering basic cell}

The harmonic sequences selective filtering basic cell (SFBC) presented in [6] [7] (some of its considerations are reproduced in this paper) performs the mathematical operations shown in Fig.1. The signal used for modulating and demodulating is, in this case, the positive sequence associated with the selected frequency $w_{c}$. The filters used for the $\mathrm{p}$ and $q$ signals are low pass filters with equal $\lambda$ gains. The obtained currents have only the harmonic positive sequence of the input current but with inverted sign. These results can be generalized for the negative and zero sequences. (In case of the presence of zero sequences, it should be better to eliminate them by using properly connected transformers instead of a four branch inverter in the active filter). A control scheme which compensates the load current is shown in Fig. 2. Finally, Fig. 3 represents SFBC and 


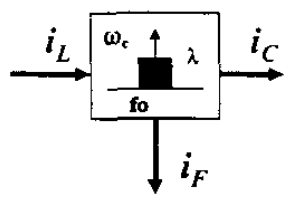

Fig. 3. Selective filtering basic cell: SFBC

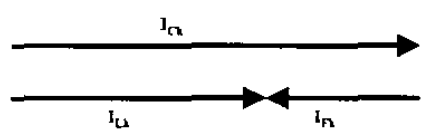

Fig. 4. Harmonic sequence compensation

also defines the sign convention which has been adopted in the electric node where the active filter is connected.

Suppose that the load current $i_{c}$ has a $k$ harmonic sequence current with rms value $I_{C k}$. Then, the filter current $i_{f}$ will drain. from the line current which will be compensated, a current that will only have such $k$ harmonic sequence. Its rms value is

$$
I_{F k}=\lambda_{k} I_{C k}
$$

Considering the electric node

$$
i_{L}(t)=i_{C}(t)+i_{F}(t)
$$

and taking into account the sign change in the schema of Fig.1,

$$
I_{L k}=I_{C k}-I_{F k}
$$

where $I_{L k}$ is the rms value of the phase vector of the current of the harmonic sequence under consideration. Therefore, the active filter current has a rms value which is modulated by the gain $\lambda_{k}$ and a sign which is opposed to the sign of the current to be compensated (Fig. 4).

\section{B. Optimum filtering}

The regulations establish a certain THD. If the regulations text is to be strictly followed, the three phases of the electric system should meet that requirements in steady state. The expression for the phase $\mathrm{R}$ current is

$$
i_{R}(t)=\sum_{k=1}^{\infty} \sqrt{2} I_{R k} \sin \left(w_{k} t+\delta_{R k}\right)
$$

Taking into account the expressions of the hammonic currents of each phase in function of the positive and negative sequence phase vectors [15] [16] and assuming that there are not zero sequence components, then

$$
\begin{aligned}
i_{R k}= & \sqrt{2} I_{+k} \sin \left(w_{k} t+\delta_{+k}\right)+ \\
& \sqrt{2} I_{-k} \sin \left(w_{k} t+\delta_{-k}\right) \\
i_{S k}= & \sqrt{2} I_{+k} \cdot \sin \left(w_{k} t+\delta_{+k}-\frac{2 \pi}{3}\right)+ \\
& \sqrt{2} I_{-k} \sin \left(w_{k} \cdot t+\delta_{-k}+\frac{2 \pi}{3}\right) \\
i_{T k}= & \sqrt{2} I_{+k} \sin \left(w_{k} \cdot t+\delta_{+k}+\frac{2 \pi}{3}\right)+ \\
& \sqrt{2} I_{-k} \sin \left(w_{k} \cdot t+\delta_{-k}-\frac{2 \pi}{3}\right)
\end{aligned}
$$

Considering that PASSIVE sequences will not be affected. because $i_{C}=i_{L}$ for them,

$$
\sqrt{I_{E}^{2}-\sum_{P} I_{C_{p}}^{2}}=\sqrt{\sum_{H} I_{L h}^{2}}=I_{c}
$$


where $I_{o}$ is defined as the controllable residue. Taking into account equation (3) results

$$
I_{o}=\sqrt{\sum_{H}\left(I_{C h}-I_{F h}\right)^{2}}
$$

On the other hand, it can be seen that in the worst case, the maximum instantaneous current that can be obtained in one phase is given by the expression

$$
i_{F M A X}=\sum_{H} \sqrt{2} I_{F h}
$$

Then in order to obtain an active filter of minimum current, the currents $I_{F h}$ which verify (15) and minimize (16) must be determined. In order to apply the Euler-Lagrange minimization method the following definitions has been made

$$
\begin{aligned}
& I_{C}=\left[I_{C 1} \ldots I_{C M}\right]^{t} \\
& I_{F}=\left[I_{F 1} \ldots I_{F M}\right]^{t} \\
& W=[1 \ldots 1]^{t} \quad W^{t} \Psi^{-}=M
\end{aligned}
$$

where $M$ is the number of harmonic sequences that are being modified. Using this notation. (15) and (16) can be written as

$$
\left(I_{C}-I_{F}\right)^{t}\left(I_{C}-I_{F}\right)-I_{0}^{2}=0 \quad i_{F \max }=\sqrt{2} W^{t} I_{F}
$$

hence the lagrangian is

$$
\lambda=W^{t} I_{F}+\zeta\left[\left(I_{C}-I_{F}\right)^{t}\left(I_{C}-I_{F}\right)-I_{0}^{2}\right]=0
$$

The solution is obtained when

$$
\frac{\delta \lambda}{\delta \zeta}=\frac{\delta \chi}{\delta I_{F}}=0
$$

The solution is

$$
I_{C}-I_{F}=\frac{I_{0}}{\sqrt{M}}=I_{S}
$$

where $I_{S}$ is defined as the current value to which the active filter must reduce the line currents of all the harmonics sequences that belong to $\mathrm{H}$ group. This is equivalent to say that the optimum is obtained by imposing the same relative harmonic distortion value for all the harmonic sequences that are being modified.

\section{Practical implementation}

The upper part of Fig. 5 shows a particular case where all the currents have initially a value higher than $I_{S}$. It may happen that an harmonic sequence of group $\mathrm{H}$ has originally a value which is lower than $I_{S}$. The lower part of Fig. 5 shows an example where it can be observed that for the harmonic sequences 1 and 3 . in order to obtain $I_{S}, I_{F 1}$ and $I_{F 3}$ must be injected by the filter, but compensation of $I_{C 2}$ is not necessary. So, an adjustment must be done because sequence $I_{C 2}$ must pass to $\mathrm{P}$ group of sequences that are not being modified, with the corresponding recalculation of $I_{o}$, obtaining then $I_{o}^{*}$. As a consequence a new value of $I_{S}$ is obtained, and it will be designated $I_{S}^{*}$. It can be shown that $I_{S}^{*}>I_{S}$. Fig. 5 shows how the currents $I_{F 1}^{*}$ e $I_{F 3}^{*}$ were reduced because of the contribution of $\left(I_{S}-I_{C 2}\right)$.
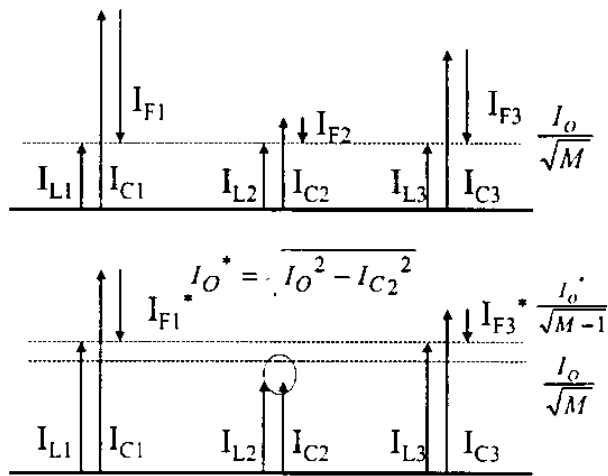

Fig. 5. Optimization in practice

Now, it can happen that as a result of applying $I_{S}^{*}$, the currents of another sequence of those to be modified, remain below this value. In that case, it would be necessary a new iteration step which consists in considering this sequence as belonging to the $\mathrm{P}$ group. This procedure should be followed, until no more harmonic sequences could change from group $H$ to $P$. If all the harmonic sequences finally appears as belonging the $P$ group, it can be shown that the required harmonic distortion was initially verified and the active filter was not necessary.

\section{And the individual maxima?}

The regulation establishes also a restriction for each one of the harmonic sequences. Hence, each couple $I_{L+j} I_{L-j}$ must comply with certain limit $I_{o j}$. A particular case of (13) can be written as

$$
I_{o j}^{2}=I_{L+j}^{2}+I_{L-j}^{2}
$$

On the other hand, the maximum current associated with $\left(I_{L+j}\right.$, $I_{L-j}$ ) that the active filter will drain is

$$
i_{j F \max }=\sqrt{2}\left(I_{F+j}+I_{F-j}\right)
$$

thus, the goal is to minimize (23) with the restriction

$$
I_{o j}=\sqrt{\left(I_{C+j}-I_{F+j}\right)^{2}+\left(I_{C-j}-I_{F-j}\right)^{2}}
$$

Which is the same as applying the result (15) to (21), then the solution is

$$
I_{C}-I_{F}=\frac{I_{o j}}{\sqrt{M}}=I_{s j} \quad M=2
$$

the solution is shown in Fig. 6. It could happen that again any of the harmonic sequences could be less than $I_{s j}$ from the beginning so the new $I_{s j}^{*}$ must be calculated in the way which has aiready been described.

As a summary, the calculation procedure is:

Step (a). - The limit of each harmonic frequency as a function of its specific limit ( $M$ is initially 2 ) is calculated. The first set of values $I_{L k}^{*}$ is obtained. 


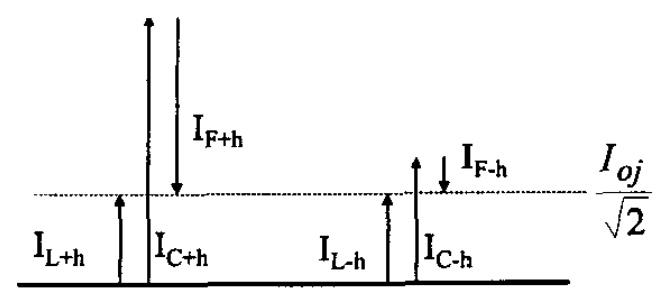

Fig. 6. Individual harmonic optimization

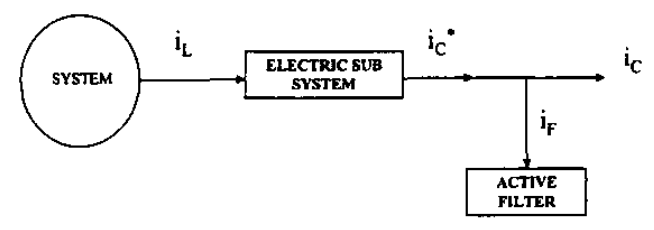

Fig. 7. Generic system

Step (b).- Taking $I_{C k}$ as the calculated values $I_{L k}$ from Step (a), the compensation for complying with the $T H D_{p}$ criteria is calculated. The harmonic sequences which it is not necessary to compensate are separated from the $H$ set and included in the P set and a new $I_{L k}^{*}$ is determined. Then $I_{S}$ is calculated again. This iterative process must be continued until there are not sequences to be separated from the $\mathrm{H}$ set. Then a final $I_{L k}^{* *}$ is calculated. $I_{L k}^{* *}$ are the values that the line current must take because of the active filter.

Step (c).- For each one of the sequences the gain of the low pass filter of each SFBC is calculated. Taking into account that those gains satisfies (1) and bearing in mind the expression (3) the gain $\lambda_{k}$ is obtained

$$
\lambda_{k}=\frac{I_{C k}}{I_{C k}-I_{L k}^{* *}}
$$

It must be noticed that a particular harmonic sequence which is corrected in step (a) can remain unchanged in step (b). Also, it could happen that after applying step (a) it is not necessary to compensate in accordance to step (b). In this last case, the total distortion goal is accomplished in excess. However, if all the harmonics required by the regulations are modified in step (a), in such a way that all of them verify exactly the allowed maximum, the total distortion limit goal will not be accomplished, and step (b) should be applied. The reason is that the regulations establish a lower THD than the one that results from the individual harmonic distortion requirements.

\section{REMOTE SELECTIVE ACTIVE FILTER}

In the general case (Fig. 7), it may be necessary to meet the regulation requirements at a place which is different from the location where current compensation can be carried out practically. A steady state transference can be established between this two points of the electric system, hence

$$
I_{L k}=q_{k} I_{C k}^{*}
$$

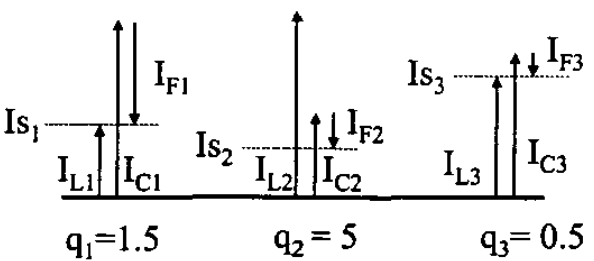

Fig. 8. Remote optimization example

where $q_{k}$ is the steady state transference. This equation links the currents values $I_{L k}$ and $I_{C k}^{*}$. Applying to (27) the same procedure which was used to obtain (14) and (15), the equivalent restriction for this case is

$$
I_{o}=\sqrt{I_{E}^{2}-\sum_{P} q_{p}^{2} I_{C p}^{2}}=\sqrt{\sum_{H} q_{h}^{2}\left(I_{C h}-I_{F h}\right)^{2}}
$$

The value to be minimized is still the one expressed in (16). The same optimization method Euler-Lagrange will be applied. The weighted matrix $Q$ is

$$
Q=\left[\begin{array}{ccc}
q_{1}^{2} & 0 & 0 \\
0 & \cdots & 0 \\
0 & 0 & q_{M}^{2}
\end{array}\right]
$$

Using the vectors $I_{C}, I_{F}$ y $W$ which were already defined, (28) changes to

$$
\left(I_{C}-I_{F}\right)^{t} Q\left(I_{C}-I_{F}\right)-I_{0}^{2}=0
$$

Then the lagrangian will be

$$
\chi=W^{t} I_{F}+\zeta\left(\left(I_{C}-I_{F}\right)^{t} Q\left(I_{C}-I_{F}\right)-I_{0}^{2}\right]=0
$$

imposing (20) the solution is

$$
I_{C}-I_{F}=\frac{Q^{-1} W}{\sqrt{W^{t} Q^{-1} W}} I_{0}
$$

then for each one of the harmonic sequences that have been modified by the active filter, (32) can be expressed as

$$
I_{C h}-I_{F h}=\frac{\frac{1}{q_{h}^{2}}}{\sqrt{\sum_{H} \frac{1}{q_{h}^{2}}}} I_{o}=I_{S h}
$$

This result which is similar to (21), introduces the weighting concept, relative to the grade of propagation of each harmonic in the electric system. In the particular case that $q_{h}=1 \mathrm{Vh}$, the result is the same as in (21). The practical calculation procedure to determine the current level that each harmonic sequence must reach, is similar to the one described in section II-C. However, in this case there is not a single value $I_{S}$; each harmonic sequence has a corresponding $I_{S h}$. For negative and positive sequences of a determined frequency the same value of $q_{h}$ is used. Fig. 8 shows an example with three harmonic sequences with their corresponding levels $\left(I_{S}\right)$ and gains $q_{h}$. 


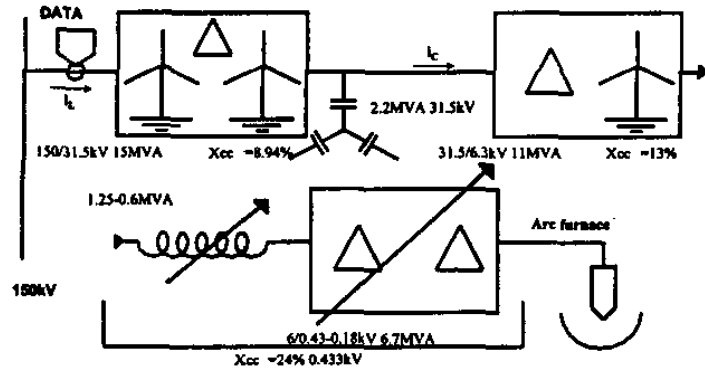

Fig. 9. Studied real system

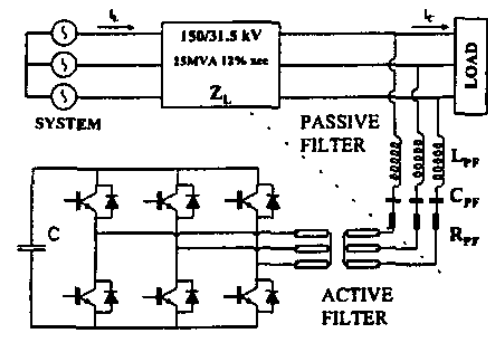

Fig. 10. Hybrid active filter connected at $31.5 \mathrm{kV}$

\section{REsults}

The complete study for a real case was presented in [7]. The original system is the one shown in Fig. 9. To meet the regulations at $150 \mathrm{kV}$, an hybrid shunt filter connected at $31.5 \mathrm{kV}$ is installed using an existent 2.2 MVA capacitor. The impedance $Z_{L}$ of Fig. 10 is the $150 / 31.5 \mathrm{kV}$ transformer shortcircuit impedance. In this case the passive system transference is

$$
\frac{i_{L}}{i_{C}}=\frac{1}{1+\frac{Z_{L}}{Z_{P F}}}
$$

where $Z_{P F}$ is the series connection of $L_{P F}, C_{P F}$ and $R_{P F}$. That transference is shown in Fig. 11. Notice that $Z_{P F}$ is tuned in $8.8 f_{1}\left(f_{1}\right.$ is the fundamental frequency) and the resonance $Z_{L}$ is located in the interharmonic $6.5 f_{1}$.

Fig. 12 shows the resulting filter gains $\lambda_{k}$. The optimization was performed with a value for $T H D_{p}=12 \%$ and with the maximum allowed individual values which are shown in Fig. 15. For example, in the initial current, the third harmonic to be compensated has a negative sequence which is higher than the positive one. In that case, through the optimization procedure, the negative sequence is filtered until it equals the positive one. Since this is not enough, some additional filtering must be performed. In the same way, positive and negative sequences of the sixth harmonic must be compensated almost $80 \%$ to oppose to the resonance that has been already mentioned. Finally, it is not necessary to compensate the $8^{\circ}, 9^{\circ}$ and $10^{\circ}$ harmonics because of the tuned filter $Z_{P F}$ effect. Applying a filter with $\lambda_{k}$ gains to the initial currents prior to compensation (Fig. 13), simulation results shown in Fig. 14 and 15 are obtained. As a calculation

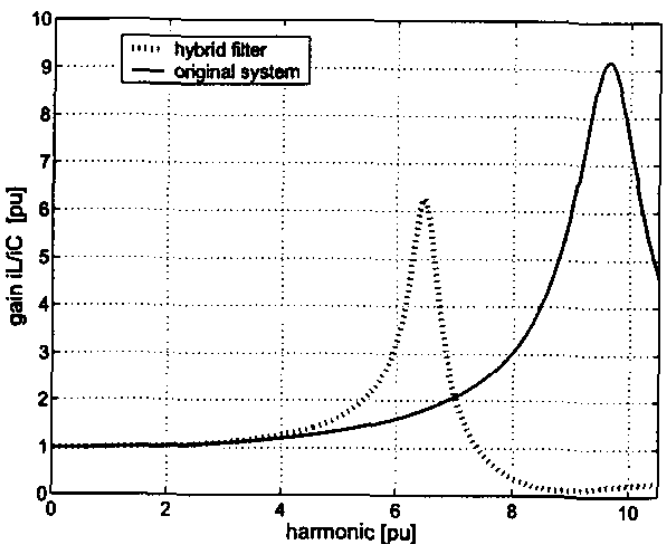

Fig. 11. Original and hybrid passive transference $i_{L} / i_{C}$.

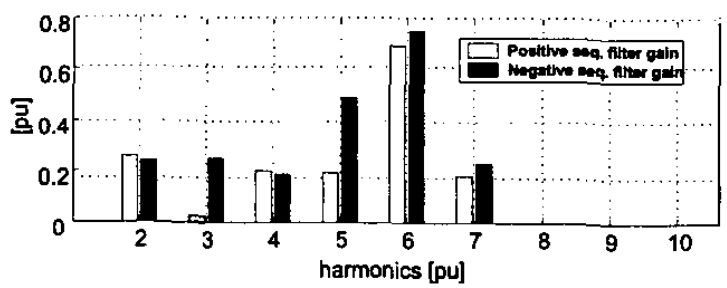

Fig. 12. Optimum gains $\lambda_{k}$. Positive sequences to the left and negative to the right

strategy the SERIES method was used [6] [7] were the output of a SFBC is the input of another SFBC. The final distortion of current $i_{L}$ comply with the established value $T H D_{p}$ and also with the allowed maximum limits. Although the real system has a non periodic characteristic, a good response in real time can be obtained. The simulated results of installing an entirely active shunt filter at $150 \mathrm{kV}$ bus bar and applying the same optimization criteria, have been presented in [6]. Fig. 16 shows that those results are worse than those obtained by using an hybrid active filter at $31.5 \mathrm{kv}$ bus bar. The current values are similar, but the active filter has to handle the $8^{\circ}, 9^{\circ}$ and $10^{\circ}$ harmonics which - in the hybrid compensation - are attenuated by the resonant filter $Z_{P F} 0$. Furthermore, although the hybrid filter must handle the whole fundamental reactive current, the voltage at the inverter terminal will be lower.

\section{CONCLUSIONS}

A new methodology for the calculation of the different SFBC gains of a selective active filter in order to comply with certain harmonic emission regulation has been presented. Since it was needed to apply this strategy to a remote compensation schema, the methodology was also extended to that case. The simulation results show that although the method was developed for steady state, it can be applied in real time and the obtained results shows a fair agreement with comparable to those expected.

For the studied particular system, two compensation alternatives were compared: compensating with a entirely active filter 

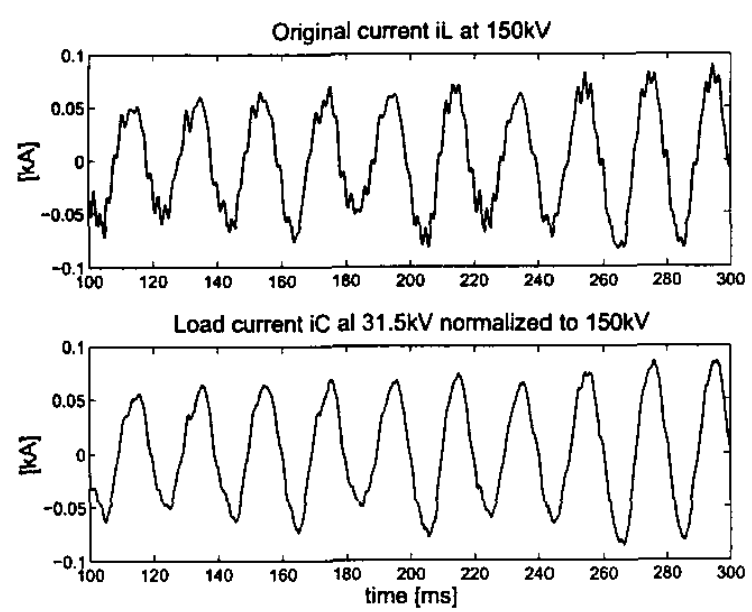

Fig. 13. Original records of the line current $i_{L}$ and load current $i_{C}$

Active Filter current if at $31.5 \mathrm{kV}$ normalized to $150 \mathrm{kV}$

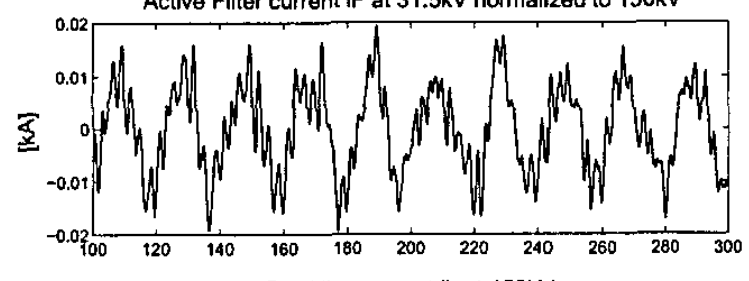

Final line current iL at $150 \mathrm{kV}$

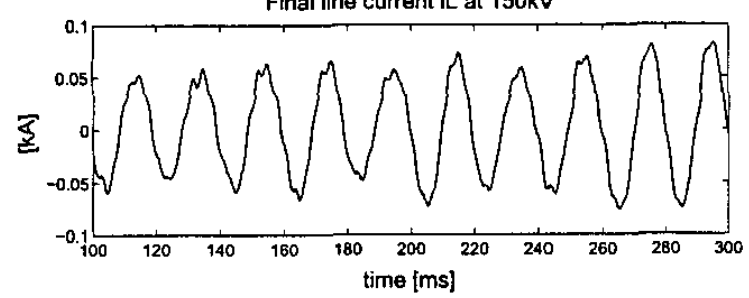

Fig. 14. Final results for filter current $i_{F}$ and line current $i_{L}$

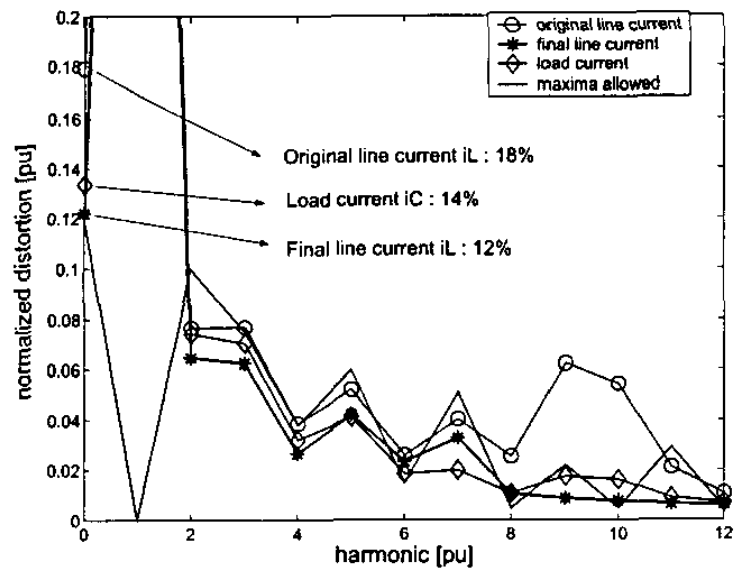

Fig. 15. Figs. 13 and 14 current spectra with permitted maxima. At $0 \mathrm{~Hz}$ is

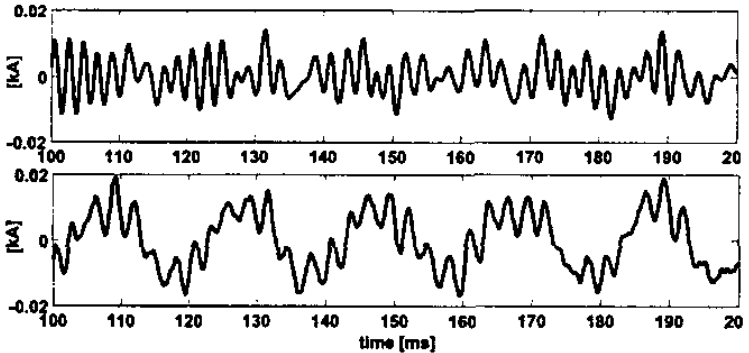

Fig. 16. Above: Current $i_{F}$ with an active filter in $150 \mathrm{kV}$ bus. Below: Current $i_{F}$ with hybrid active filter at $31.5 \mathrm{kV}$ bus bar. Nomalized to $150 \mathrm{kV}$

located at $150 \mathrm{kV}$ bus bar or compensating with an active hybrid filter at a remote place, downstream, at the $31.5 \mathrm{kV}$ bus bar. The comparison shows that remote compensation is an alternative to be seriously considered. The results are similar to those obtained with local compensation and the cost may be substantially lower.

\section{REFERENCES}

[1] H. Akagi, Y. Kanazawa, and A. Nabae, "Instantaneous reactive power compensator comprising switching devices without energy storage components," IEEE Trans. Ind. Appl., vol. Vol. 20, no. 3, pp. 625-630, 1984.

[2] Ente Nacional Regulador de la Electricidad Argentina, "Decreto 99/97," 1997.

[3] "Std S19 - recommended practices and requirements for harmonic control in electrical power systems," Tech. Rep., IEEE, 1992.

[4] "IEC 61000-3 electromagnetic compatibility," Tech. Rep., International Electrotechnical Commission, 1996.

[5] G. Casaravilla, C. Briozzo, and E. Watanabe, "Filtro activo de mínimo costo ajustado a la carga de un homo de arco y a las regalamentaciones sobre emisión armónica aplicables," XIII CBA-Congresso Brasileiro de Automática, pp. 1108-1113, 2000.

[6] G. Casaravilla, A. Salvia, C. Briozzo, and E. Watanabe, "Estrategias de control de filtros activos shunt selectivos de conientes armónicas," COBEP - 6 Congresso Brasileiro de Electrónica de Potencia, vol. Vol. 2 , pp. 432-437, 2001.

[7] G. Casaravilla, A. Salvia, C. Briozzo, and E. Watanabe, "Selective active filter applied to an arc furnace adjusted to hamonic emission limitations," in IEEE / PES TyD Latin America, 2002.

[8] S. Bhattacharya, P. Cheng, and M. D. Divan, "Hybrid solutions for improving passive filter perfomance in high power applications," IEEE Transactions on Industry Applications, vol. 33, no. 3, pp. 732-747, 1997.

[9] S. Bhattacharya and D. Divan, "Design and implementation of a hybrid series active filter system." IEEE, pp. 189-195, June 1995.

[10] V.S. Ramsden D. Basic and P. Muttik, "Hybrid filter control system with adaptive filters for selective elimination of harmonics and interharmonics," IEE Proc.-Electr. Power Appl., vol. 147. no. 3, pp. 295-303, May 2000.

[11] S.Park J.H.Shung and K.Nam, "New hybrid parallel active filter configuration minimising active filter size," IEE Proc. Electr. Power Appl., vol. 147, no. 2, pp. 93-98, Mar. 2000 .

[12] P. Mattavelli, "A closed-loop selective harmonic compensation for active filters," IEEE Transactions on Industry Applications, vol. 37, no. 1, pp. $81-89,2001$.

13] Hirofumi Akagi, "Applications of power electronics to power systems in japan," COBEP'97, Brazil, 1997.

[14] K. Al-Haddad B. Singh and A. Chandra, "A review of active filters for power quality improvement," IEEE Transactions on Industrial Electronics, vol. 46. no. 5, pp. 960-971, Oct. 1999

[15] Mauricio Aredes, Acrive power line conditioners, Ph.D. thesis, Technical University of Berlin, 1996.

[16] J. Monteiro, "Filtros hibridos Ativo/Passivo de potência: Modelagem no domínio da freqüência," M.S. thesis, COPPE - UFRJ, 1997.

$T H D_{p}$ 